\title{
Understanding and Analyzing Chat in CSCL as Reading's Work
}

\author{
Alan Zemel, Murat Cakir \& Gerry Stahl \\ Drexel University, Philadelphia, USA \\ Email: ARZ26@drexel.edu, MPC48@drexel.edu, Gerry.Stahl@drexel.edu
}

\begin{abstract}
Synchronous communication using text chat-often combined with a shared whiteboard-is increasingly used in CSCL. This form of interaction and learning in small online groups of students presents novel challenges, both for the participating students and for researchers studying their work. Chats differ from talk-in-interaction since the composition, posting and visual inspection of text and graphical objects by any given actor is not observable by the other participants. These structural constraints on the organization of interaction require that actors deploy alternative procedures for achieving what turn taking achieves in talk-in-interaction. This paper describes how communication is organized in text chat, where postings have to provide instructions on how they are to be read. This organization is contrasted with turn taking in face-to-face communication. The notion of "reading's work" provides a guiding thread, which is explicated.
\end{abstract}

Synchronous text chat seems to offer a particularly effective medium for joint learning activity, but it presents challenges for students using it because the group sense-making strategies to which they are accustomed in faceto-face interactions either do not work or work differently in the online setting, for reasons to be discussed. Similarly, sources of evidence normally available to educational researchers are not available from synchronous online interactions. Student groups develop methods of building their postings to convey instructions for how they are to be read. Chat researchers as well as chat participants must learn to pay heed to such instructions. As we will see, such instructions often involve the sequentiality of postings and the references among those postings (Sarmiento \& Stahl, 2008).

We have explored the use of text chat for discussions of mathematics in small groups of students for the past six years in the Virtual Math Teams Project (VMT) (Stahl, 2009). Although we have reported on the technology design (Stahl, 2008) and on various case studies (e.g., Stahl, 2006; Zhou, Zemel \& Stahl, 2007), this paper is our first attempt to describe the systematic workings of text chat, building on previous publications that began to clear the ground for this. We call this description a "simplest systematics" in analogy to the seminal paper on turn taking in talk by the pioneers of ethnomethodology and conversation analysis (Sacks, Schegloff \& Jefferson, 1974), which we take as our inspiration.

\section{Interacting Through Text Chat}

In CSCL online chat systems like VMT, participants can engage with each other in a variety of ways. Rather than interact through emergent talk and observable embodied action, they: exchange text postings through chat technology, post text or graphic elements on a virtual whiteboard and/or use referential tools provided by the system.

Interaction in VMT involves actors using computer hardware and software in ways that allow for the production of shared, displayed representations or virtual objects possessing various features that allow these objects to serve as the means by which participants interact. Participants are represented in various ways in VMT in terms of various conventions and practices of action identification. These representations-i.e., naming conventions and displays, avatars, authored messages, posted graphical objects, etc., as well as various changes in the appearance of objects or the state of the system - provide documentary evidence of actor presence (Zhao, 2003) and engagement with the system. It is these same resources that are put to work to constitute social interaction among actors in a chat. In other words, it is through the mediated exchange of what can be seen as locally relevant textual and graphical resources that chat participants organize and constitute their interaction. The problem that chat participants face in task-directed chats of the sort we inspect is to coordinate their participation to collectively and collaboratively perform the task with the technical resources available in the hardware and software and with the textual and graphical resources they construct as relevant to their ongoing tasks. As it happens, this is a challenging problem that involves the management of and allocation of attention across multiple interface areas of the chat system and the ability to produce domain relevant artifacts (text messages, graphical artifacts, etc.) for inspection by others participating in the exchange of such artifacts.

Because these systems are designed in ways that allow participants to produce and inspect visual artifacts in particular ways, a natural question arises as to the nature of interaction that emerges in such environments. How do these interactions differ from talk-in-interaction? Speech exchange systems, like face-toface conversation, telephony, video conferencing, etc., exploit and are constrained by the technical affordances of speech production. As Sacks, Schegloff \& Jefferson (1974) described, speech exchange systems rely on the 
affordances of the technology of talk to organize social interaction. The sequential organization of face-to-face conversational speech exchange is a product of the fact that actors are co-present to each other in an embodied way, which necessitates taking turns at listening and speaking. Thus actors allocate opportunities to speak and to listen in various ways such that one speaker speaks at a time and they repair problems of intelligibility that arise from mishearings, poorly produced speech and overlapping speech.

Chat environments, on the other hand, are not speech-exchange systems at all, but rather systems of interaction that involve the display and inspection of visual artifacts, including texts (Garcia \& Jacobs, 1999). The sequential organization of the production of visual artifacts is observable, available and documentable-and is something to which chat participants orient in their ongoing engagement in and through chat. However, the sequential organization of chat is not based on the same considerations that govern the sequential organization of talk-in-interaction (Garcia \& Jacobs, 1999). One obvious difference is that overlap can happen in talk but cannot happen in most kinds of chat systems. Overlap is a phenomenon of talk in interaction. Problems of hearability, problems related to the allocation of turns in talk, problems that provide for repair in talk in interaction simply do not occur in chat. Different kinds of interesting troubles with respect to the intelligibility of postings can and do occur in chat, but these have to do with sequential placement of postings and other displayed graphical artifacts. It is because of this and other differences in the technical production possibilities afforded by chat systems that we feel compelled to provide the beginnings of a simplest systematics of online chat and to describe some of the ways that interactions through online chat differ from interactions through speech.

\section{Co-Presence}

The analysis we present involves consideration of a number of foundational features that are constitutive of social interaction. According to Goodwin (2000):

The accomplishment of social action requires that not only the party producing an action, but also that others present, such as its addressee, be able to systematically recognize the shape and character of what is occurring. Without this it would be impossible for separate parties to recognize in common not only what is happening at the moment, but more crucially, what range of events are being projected as relevant nexts, such that an addressee can build not just another independent action, but instead a relevant coordinated next move to what someone else has just done. (p. 1491)

Not only must participants recognize what is happening, but participants must recognize "in common" what is happening. This notion strongly ties to Pollner's (1974) notion of mundane reasoning and Hanks' (2000) notion of indexical symmetry. Central to Goodwin's description are the practical achievements of presence, copresence and the shared recognition of "what is occurring" in the scene. In other words, interaction arises when actors act in coordinated ways through mutual engagement with respect to recognizable and meaningful activities and shared-in-common and mutually recognizable orientations to (1) each other, (2) their actions and (3) features of the scene in which these activities are occurring. While Goodwin talks about coordinating contiguous actions as relevant to interaction, it is necessary to recognize that contiguity of action is not a requirement in all systems of social interaction.

In addition, social interaction requires more than reciprocal contact. Interaction requires co-presence. Co-presence is a condition of and for social interaction. According to Zhao (2003, p. 446), co-presence is "a form of human colocation in space-time that allows for instantaneous and reciprocal human contact." In ethnomethodological terms, co-presence is a gloss for the notion of a shared intersubjective world and the shared sense-making and reasoning practices by which shared inferential practices manifest and sustain the reality of that intersubjective world (Pollner, 1974). In short, social interaction requires reciprocity of perspectives founded in a common life-world that allows participants to act as though each is seeing what the other is seeing despite any differences in perspective that might arise (Pollner, 1974). According to Hanks $(2000$, p. 7), reciprocity of perspective is "neither similarity ('sharedness'), nor congruence per se, but the idea that interactants' perspectives are opposite, complimentary parts of a single whole, with each oriented to the other." It provides the basis by which an actor can reliably act as though other actors can, to some degree, see what she sees, know what she knows, feel what she feels, etc.

The more interactants share, the more congruent, reciprocal and transposable their perspectives, the more symmetric is the interactive field. The greater the differences that divide them, the more asymmetric the field. (Hanks, 2000, p. 8).

This reciprocity of perspectives establishes a sense of co-presence in which the experiences and perceptions of the actors in a scene become practically available to each other. The practical problem for actors engaged in online chat is quite simply to figure out how to use the visual artifacts (virtual objects and text) and the affordances of the chat system so that they and others can recognize these artifacts and their use as constitutive of social interaction in that environment. 


\section{Interaction as Reading's Work}

It is clear from the data we have inspected in the VMT Project that chat systems display an alternative organization of social interaction, one that is not based on the notions of consequential contiguity of action and turn taking in conversation. Specifically, in VMT actors may compose and post texts, develop and post graphical objects, etc., without being constrained by the actions of others precisely because the system allows it and because those actions are not witnessed or witnessable by other chat participants. In conversation, turn taking arises from just this notion that the witnessed and witnessable production of talk constrains the talk of others. The nature of these constraints is what organizes action into turns, turn sequences and the like. Thus turn taking requires that an actor and the recipients of that actor's actions collaborate to allocate their participation in orderly ways to produce meaningfully contiguous actions (Schegloff, 2007). Online chats often seem confusing and disorderly (Fuks, Pimentel \& Pereira de Lucena, 2006; Herring, 1999) precisely because there is no obvious way to achieve the same kind of orderly contiguity as can be achieved in talk-in-interaction.

In practice, the achievable orderliness of online chat interactions is produced not by the way participants collaborate to produce actions, but by readers who, through the work of "reading," are responsible for identifying the progressively sequential nature of observable online postings even though the procedures of turn-taking in a strict sense cannot apply. One oft-heard complaint about chat is that postings are often "out of turn" (Garcia \& Jacobs, 1999), which causes participants to struggle with the continuity or, as Schegloff (2007) calls it, the progressivity of ongoing interaction.

Contiguity does not operate in chats with the same manner as in talk-in-interaction. The actions participants perform to produce text or graphical objects for display and distribution to others are not observable or available to anyone but the person performing those actions. Anyone can post a text or a graphical object at any time without regard for the actions of others. This is a feature and affordance of common chat systems. Thus, any sense of progressivity and turn organization can only be achieved ex post facto as recipients' work of inspecting postings for how they could be constituted as a sequence of actions. Contiguity is problematic as a basis for establishing and recognizing the sequential organization of postings in chat. Consequently actors resort to other procedures and resources to achieve a sense of progressivity in their chats.

The constitution of sequentiality and the perceived orderliness of chat interaction is a reader's achievement in chat. The work required to make sense of textual and graphical postings is what Livingston terms reading. According to Livingston (1995), texts are built in ways that inform the reader how to read them. While Livingston's notion of reading is oriented to text-based materials, we would suggest that a more general notion of reading would involve the work of making sense of visual artifacts whether they are text-based, graphical, etc. Actors who are working to make sense of graphical or textual artifacts assume that these artifacts are produced, organized and displayed for inspection and to inform and instruct viewers concerning how they are to be understood. In other words, each visual artifact provides clues for how viewers are to make sense of it and, in the case of VMT, for how they are also to make sense of that artifact in relation to previously posted graphical artifacts and previous chat postings.

\section{Interaction's Traces}

The data we inspect for our analysis of social interaction in online chat consist of time-stamped chat logs of math problem solving in the VMT Project, where groups of three to five students in grades 6 to 11 collaborated online to solve math problems that required reflection and discussion. Each session lasted an hour and was supervised by a VMT facilitator who did not participate in problem-solving work with the other participants. The students understood that they were to collaboratively work together to produce solutions to posted math problems. This was made evident in the way that they managed their participation in the chats.

Various software platforms were used to facilitate these sessions, including AOL's Instant Messenger (AIM) and versions of a custom VMT chat environment. AIM provides a simple chat interface where the users interact with each other by exchanging short texts. These sessions were recorded as chat transcripts with participant identifier, the time-stamp of the posting and the content posted (see Log 1). Note how these postings use many textual features to guide the work of reading them (words, math symbols, chat abbreviations, capitalization, ideographic conventions, etc.); these guides are available in the log traces just as they were in the live postings.

$\log 1$.

pin (8:40:42 PM):

pin (8:40:46 PM):

pin (8:40:52 PM):

pin (8:40:55 PM):

pin (8:40:58 PM):

Avr (8:41:03 PM):

\author{
this is easy \\ for the 12 triangle \\ $144=36+x$ \\ so $x=/ / / /$ \\ $\ldots$...* \\ NOBODY DO THE MATH
}




$\begin{array}{lll}7 & \text { Avr (8:41:06 PM): } & \text { I'M DOING IT } \\ 8 & \operatorname{pin}(8: 41: 12 \text { PM): } & \text { square root 108 } \\ 9 & \text { Avr (8:41:16 PM): } & \text { I KNOW I KNOW } \\ 10 & \text { Sup }(8: 41: 19 \text { PM): } & \text { Iol } \\ 11 & \text { Avr }(8: 41: 20 \text { PM): } & \text { LET ME DO IT } \\ 12 & \operatorname{pin}(8: 42: 04 \text { PM): } & \text { be my guest } \\ 13 & \text { Avr (8:42:39 PM): } & \text { okay }\end{array}$

In contrast to AIM, the VMT environment provides two interactive components, namely a text-based chat and a shared whiteboard. One of the unique features of the VMT system is a referencing support mechanism that allows users to visually connect their chat postings either to previous chat postings or to areas on the whiteboard. VMT chat sessions are also recorded as transcripts with participant identifier, the time-stamp of the action performed and the content posted. Due to the added complexity of the whiteboard component and the referencing tool, VMT transcripts include additional types of actions, such as drawings, manipulation of an object on the board, messages indicating start/end of typing activity, referencing pointers.

In an effort to tackle the practical challenges of analyzing such complex transcripts we used the VMT Replayer tool, which allows us to replay a VMT session as it unfolded in real time based on the time-stamps of actions recorded in the log file. The order of actions we observe with the Replayer as researchers exactly matches the order of actions experienced by the users.

\section{Technologically-Mediated Social Interaction}

Interactants in chat work with chat technology as a form of technologically mediated social interaction. Technically (from the perspective of the network technology), interaction in chat-only systems is achieved as the posting of texts to the chat system for distribution to all the nodes logged into the chat server so that other participants have the opportunity to view the posted texts, read them and respond. For example, it is understood by users of chat systems that texts posted within a chat interface are made available to other participants and that other participants are to orient to these postings in their subsequently posted texts.

Even when a text is posted to which no one responds, the absence of a response may be a meaningful and consequential social action. For example, if a text is posted and no one responds, the lack of response may be treated as an accountable matter. Even if no account of a lack of response is called for, the posting and its subsequent treatment are social facts for the participants in the chat.

In chat systems with whiteboards, participants read and produce both text postings and graphical displays. Graphical artifacts posted to a whiteboard are available for other participants to view. Objects made available for inspection in the whiteboard are often treated as referential resources for and by participants in the chat. Participants in online chats with whiteboards constitute and treat each other as readers and authors of texts and graphical objects in their interactional work. (There are, of course, features of the interactional work that are oriented toward the management and use of the technology itself, which occur at individual terminals connected to the chat system and which are often times not available for inspection by other participants). The consequence of this for participants and observers of chat interactions is that the sequence, organization and textual resources of chat postings and the whiteboard positioning, manipulation and semiotic resources of graphical displays constitute the indexical ground (Hanks, 1992) by which the sense-making work of chat interaction is achieved.

Typically, different areas of the user interface are devoted to whiteboard activity and to chat. Participants are faced with the challenge of monitoring different areas of the interface while at times also producing text or graphical artifacts for posting and display. Participants appear to orient to the fact that simply posting a text message or a graphical artifact may not always be adequate to assure that other users will "see" it or give it the consideration that the author might hope for. Because a participant's attention may not be given to that part of the interface displaying a newly posted text or graphical artifact, the producer of a text or graphical artifact cannot be sure that any given recipient is aware of a posted text or artifact unless an explicit response to that posting is produced and displayed. While graphical displays in the whiteboard are viewable by any participant, such displays need not necessarily be designed or produced to solicit responses from others, and they are typically not treated that way (although on occasion they are). Whiteboard items are often treated as displays to which participants orient in the production of chat messages. They are treated as illustrations of conceptual objects that are available for inspection, but they are not used specifically to elicit responses from viewers. Such responses are elicited through chat postings that make reference to these items. The whiteboard postings serve to provide indexical ground for chat postings. While user-generated text postings in the chat area are oriented to, produced and treated as a way of soliciting in-kind responses from others, whiteboard postings are typically oriented to, produced and treated as ways of establishing indexical symmetry (Hanks, 1996). 
Thus there are significant differences between posted text messages and other graphical artifacts made available in VMT. These differences are significant because users of VMT themselves find the differences relevant and orient to these differences in their ongoing interaction. Furthermore, designers of CSCL chat systems recognize, orient to and display the significance of these differences in the way that these systems are designed. For example, in the VMT system, chat activities occur separately from the exchange or display of visual artifacts on the whiteboard. Different technologies are deployed to handle the exchange and display of graphical and textual artifacts. Furthermore, user interfaces (viz., chat and whiteboard) are designed to reflect these differences. Therefore, as we develop this analysis, we distinguish and demonstrate the relationship between two categories of visual artifacts, i.e., text postings (or messages) to the chat interface and graphical displays on the whiteboard.

The data we examine systematically demonstrate that text exchange through chat is used as the principle method of achieving "real-time" social interaction among participants. Progressivity and the appropriate projection and production of in-kind responses in chat serve as the basis by which participants come to treat their actions as social interaction. Indexical symmetry is an achievement of both chat and whiteboard activity. While text postings accumulate and scroll out of the visual field, whiteboard content is systematically used to establish indexical symmetry; relevant artifacts and occasionally emergent content are displayed for ongoing or persistent deictic reference over the course of ongoing chat interaction. In other words, whiteboard contents are items (1) which participants add and modify to display and share their then-current state of practical reasoning and/or indexical ground with respect to the task at hand and (2) to which participants refer in their ongoing chat interaction as persistent and recoverable demonstrations of practical reasoning and/or indexical ground.

\section{Text Postings in Chat}

Recent treatments of online chat interactions have documented that chats are significantly different from faceto-face interactions. In their seminal work on online chats as interactional phenomena, Garcia and Jacobs (1998; 1999) have noted that turn taking, turn allocation and repair in chat differs significantly from the way that turn taking, turn allocation and repair are performed in face-to-face interaction. The main difference is that online chats are not speech-exchange systems; rather they are text-exchange systems. It is no wonder that turn-taking organization and repair are very different phenomena than their counterparts in face-to-face interaction because the practical achievement of sequencing actions in chat is done so differently from speech by virtue of the technology of online chat. One consequence of this is, as Garcia and Jacobs (1999) point out, that the monitoring and posting of text messages are more loosely linked to the actions of other chat participants than the monitoring and execution of conversational actions among interlocutors in face-to-face interaction. Furthermore, where violations of projected next-turn actions are treated as repairable or accountable matters in face-to-face interaction, they are routinely treated as affordances of the technology by which online chats are achieved and thus do not always warrant the production of repairs or accounts. Of course, repair happens in chats, but its organization and achievement are subject to the technical constraints that govern the posting of messages (Schönfeldt \& Golato, 2003).

Text postings in chat are designed to be read by all participants in the chat. Text messages differ from speech in a number of interactionally significant ways. In most chat systems, text messages are composed "in private," i.e., only the composer can witness its production, no other chat participants see the emergent text as it is being composed". Chat participants only "see" a text after it is sent by its author. This process of text production and distribution presents participants with significant coordination concerns as they exchange texts.

One interactionally relevant consideration of online chat is that actors cannot closely coordinate with others by monitoring what others are doing since the actual production of chat artifacts (text messages, etc.) is unavailable for examination by recipients (Garcia \& Jacobs, 1999). Problems of sequentiality and coherence become relevant to participants and are managed in the way that actors design their texts to be read and recipients come to read these texts. Therefore, chat participants face the task of producing texts to be read in ways that are designed to display their sense and to read those texts in the ways they were designed to be read, even though the actual production of postings cannot be observed.

In face-to-face interaction, actors rely on the sequential organization and production of talk, of embodied action, environmental resources, etc., for the achievement of interactional sense making. In online chat, participants only have access to posted texts, which typically do not display their sequential construction, the performance of self-repairs, etc. In addition, there are no technical constraints imposed on other actors when

\footnotetext{
${ }^{1}$ Some of the earlier chat tools offered interfaces that allow their users to witness the production of messages, such as Unix Talk and earlier versions of ICQ. However, such tools need to split the screen into multiple areas dedicated to each user so that the production process can be seen at all clients. This brings scalability and intelligibility issues of the chat taking place in the environment. Now most popular chat and IM systems employ the strategy of displaying awareness messages while the user is typing, and then display the message after the user posts the message to the server.
} 
an actor composes a text. To illustrate what this means, consider the following. In speech exchange systems, when two parties speak at the same time, hearability of the speech of either party is compromised. When two parties compose and post messages at the same time, the readability of the texts is unaffected. Thus, there is no technical incentive to manage sequentiality in text-exchange systems as there is in speech-exchange systems. This doesn't mean that actors post willy-nilly in chats. Intelligibility is an issue with respect to how actors read the texts in relation to prior postings and in relation to whatever projected subsequent postings might be possibly relevant.

One example is shown in Log 2. At line 318, Avr's request, "okay can you explain how you're getting $i t$," is presented in its entirety as a completed text. We don't see it's construction. This is contrasted with the work that Pin does in lines 319 to 323, 326 and 328, where he produces a sequence of short and grammatically linked postings that constitute, as a sequence, what readers treat as an extended posting.

$\log 2$.

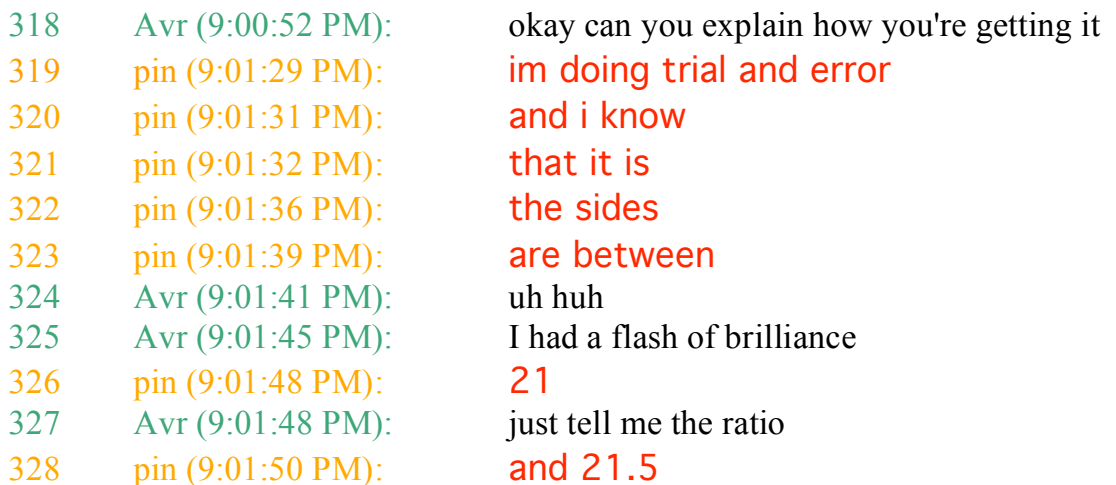

While each of Pin's postings is presented in its entirety, they are constituent elements in what is being built to be read as an extended multi-post message. By using grammatical resources and short durations between postings, Pin is able to display in the texts he is posting that they are being presented to be read as a string of connected postings. In this way, users are occasionally able to approximate the display of the sequential construction of postings.

In Log 3, Lif organizes his response to Azn's query in multiple postings in such a way that the first two postings (lines 155 and 156) project the production of a longer elaboration on his findings regarding the problem at hand (line 161).

$\log 3$.
153
154
155
156
157
158
159

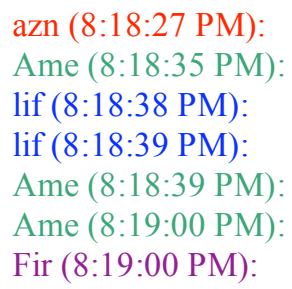

160

161

Ame (8:19:38 PM): lif (8:19:53 PM): did anyone get farther than this?

Because it never says which order the lengths of the segments are not really, all that $i$ know is that

we have to find out

I say there are six possible orders or length

well i said earlier that $i$ just used trial and error and factored it out using the number I had picked and $i$ found that it had to be less than 4

$\left(\mathrm{n}^{\wedge} 2+4+4 \mathrm{n}\right)<9<\left(\mathrm{n}^{\wedge} 2+5 \mathrm{n}\right)$ is possible

$(\mathrm{n}+2) 2<9+\mathrm{n}(\mathrm{n}+5)$ and $9<(\mathrm{n}+2) 2+\mathrm{n}(\mathrm{n}+5)$ and $\mathrm{n}(\mathrm{n}+5)<9$

$+(\mathrm{n}+2) 2$

When a participant posts a text message, it may be constructed so as to be read as incomplete, projecting that a next post by that participant (not necessarily the next post in the sequence) is to be read as a continuation of the participant's current posting. This can be done using grammatical resources such as an incomplete phrase or sentence) and other lexical resources such as ellipsis or colons.

An increasingly available feature incorporated into chat systems is the production of "awareness messages," which are system-generated indications of activity performed by others. In the systems we examine (VMT and AIM), various awareness messages were available. When an actor engaged in the composition of a text message, a system-generated message was displayed to all participants indicating that the actor was typing. Even though the awareness messages indicate that an actor is typing, recipients cannot know what is being typed until the text is posted to the system. On occasion, actors type and apparently erase their typing without posting. 
Chat repair is organized differently than repair in talk-in-interaction. Specifically, in order to effect a repair to a posted text, another text needs to be posted indicating that it is a repair and what it is repairing. This organization of repair arises because once a text is posted to the chat system, it cannot be manipulated any further. It becomes fixed even as it is displayed.

$\log 4$.

$1 \quad \operatorname{mcp}(8: 40: 15 \mathrm{PM})$

Oh, I see where your 18 and 10.125 are from now. I had already doubled and you waited till later. Yes, I'm with all this.

$2 \quad \operatorname{real}(8: 40: 31 \mathrm{PM})$ : I got it

$3 \quad \operatorname{mcp}(8: 40: 40 \mathrm{PM})$

$4 \quad \operatorname{mcp}(8: 40: 44 \mathrm{PM})$ :

$5 \operatorname{mcp}(8: 40: 48 \mathrm{PM})$ :

And dragging the sqrt(3) along would give exactly 156. 15 not 156

In Log 4, Mcp repairs his statement in line 3 by posting two more subsequent postings. In his first posting Mcp offers a new value (line 4). His next posting (line 5) establishes the relationship between the new value and the erroneous one he previously reported, and hence accomplishes the repair.

Another feature of text postings is that they are enduring in particular ways. Once a text is posted, it becomes part of the posting history and is accessible for review. It is possible to scroll backward in a chat to view previous postings. Once a text has been posted, it remains available for viewing in the history of the sequence of postings. This allows participants to examine previously posted texts that may have "scrolled" out of view over the course of their ongoing interaction.

The VMT chat system provides a referencing tool as an additional resource by which someone composing a text posting can link that posting to either a previously posted message or an object on the whiteboard. This tool provides actors with a graphical resource in designing their chat postings for linking the current posting to a prior one. Thus actors who compose texts and readers who read them need not only rely on lexical resources to indicate relationships between contiguous and non-contiguous postings.

The VMT referencing tool can also be used to link a current chat posting to an area of the whiteboard. It thus provides message designers with the means to make graphical indexical references in a manner that is somewhat analogous to the way gesture is occasionally deployed in face-to-face talk-in-interaction.

These are some of the features of text postings in chat. The interactional consequences of these features can be summarized as follows. By producing texts for display to other participants, actors are demonstrating their active presence by influencing and altering the state of the system by their actions. These very texts are not only produced to change the state of the system but are also produced to be read and to be responded to as meaningful by recipients. The meaningfulness of text postings derives from the work done by postings to establish a reciprocity of perspective between the text's author and its recipients. This is achieved using shared lexical, grammatical and textual resources and it is achieved by the exchange of postings that are treated as meaningful by participants. Thus text exchange in chat provides for a form of social interaction based on the production and reading of posted texts.

\section{Graphical Artifacts}

Graphical artifacts can be distinguished from text-based chat artifacts by virtue of the fact that:

- They are typically produced and displayed in a different part of the user interface than the chat system,

- They are designed for inspection by all participants but are rarely used to solicit text artifacts or other graphical artifacts from other participants and

- They call on recipients to make use of shared indexical ground and deictic practices different from those of chat for their intelligibility.

The work of producing graphical artifacts in the whiteboard involves designing and constructing artifacts to be seen and recognized in relation to ongoing chat postings and displayed whiteboard objects. This work, while similar to the work of producing for reading and reading text postings, displays certain particularities that derive from the technology of whiteboard artifact production. The technology of artifact production in the whiteboard of the VMT system involves the piece-wise production and arrangement of the constituent elements of the artifact. The piece-wise nature of artifact production allows recipients to witness the emergent achievement of the artifact on the whiteboard.

In addition, once posted, graphical artifacts on the whiteboard can be manipulated, altered, moved, etc. Actors can position or reposition one or a collection of such constituent elements in relation to other artifacts. They can also delete items from the current whiteboard space (though they remain available by scrolling back in the whiteboard history in the VMT system). This stands in marked contrast to text postings in the chat system that cannot be manipulated or altered in any way once a text is posted. 
Another feature of the VMT system is that there are awareness markers that indicate user actions in the whiteboard. These appear in the chat window as a series of colored squares. A square appears in the chat every time an action is performed and posted in the whiteboard (see Figure 1). These squares are color-coded and correspond to the colors assigned to users.

The sense-making apparatus invoked by the placement and display of a whiteboard artifact involves recognizing what is presented in relation to other whiteboard artifacts and to ongoing chat activity. Whiteboard artifacts become relevant to actors in a variety of ways. One use of such artifacts is to serve as an illustration of a matter that is topically relevant in chat postings. Because these artifacts are both persistent and mutable, they can serve as indexical resources that provide for symmetrical perspectives on a matter under consideration in chat. As part of an ethnomethodological study of cognitive scientists' whiteboard use during design meetings in a face-to-face setting, Suchman conjectured that "... while the whiteboard comprises an unfolding setting for the work at hand, the items on the board also index an horizon of past and future activities" (1990, p. 317). In other words, what gets done now informs the relevant actions to be performed subsequently, and what was done previously could be reproduced or reused depending on the circumstances of the ongoing activity.

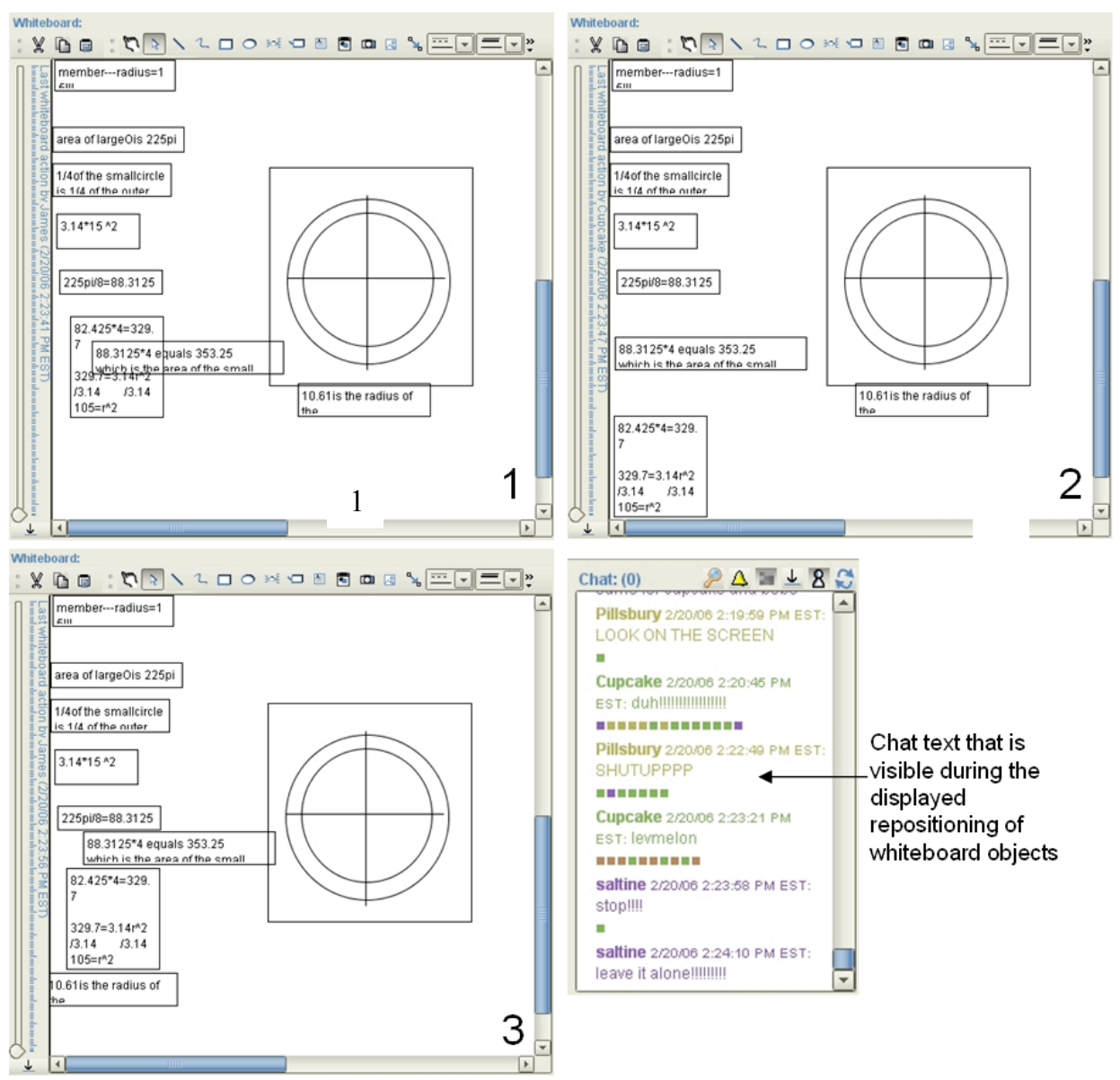

Figure 1. Movement of graphical objects to do practical reasoning.

Because of the mutable and persistent nature of whiteboard artifacts, it is possible for actors to add objects and arrange them. The production and placement of whiteboard artifacts allows an author to display to $\mathrm{him} / \mathrm{h}$ erself and other recipients the achievement of practical reasoning as the piece-wise construction of these artifacts. For instance, Figure 1 shows an example where the participants move a number of individual textboxes to achieve a particular layout on the shared space. The achieved organization displays how individual items are seen and read as related pieces of a larger organization.

Additionally, practical reasoning is demonstrated by the placement and juxtaposition of these artifacts as indexical resources relevant to the ongoing interactional work of the participants. Participants coordinate their chat activities with whiteboard artifacts and also coordinate whiteboard artifacts within the field of extant artifacts using the deictic resources of the technology (reference tools, linguistic deictics embedded in the chat, etc.) and the artifacts themselves as deictic resources. For instance, Figure 2 presents an example where a participant uses a recently completed drawing as a referential resource to formulate a question directed to his teammates: "so it has at least 6 triangles? / In this, for instance." 
Chat postings and objects posted on the whiteboard differ in terms of the way they are used as referential resources by the participants. The content of the white board is persistently available for reference and manipulation, whereas the chat content is visually available for reference for a relatively shorter period of time. This is due to the linear growth of chat content which replaces previous messages with the most recent contributions at the bottom of the chat window. Although one can make explicit references to older postings by using the scroll-bar feature, the limited size of the chat window reinforces a referential locality between postings that are visually proximal to each other. This visual locality qualifies the whiteboard as the more persistent medium as an interactional resource, although both mediums technically offer a persistent record of their contents through their scrollable histories.

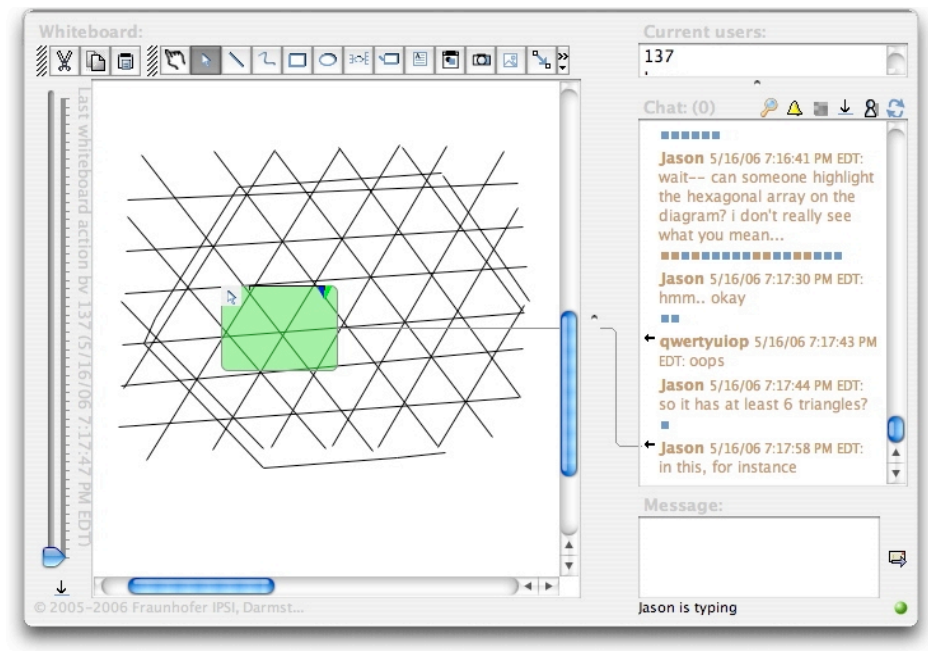

Figure 2. Jason indexes an area of the whiteboard.

\section{A Systematics of Interaction in VMT}

In this paper, we have described the systematic affordances of AIM and the VMT chat systems by which actors produce and inspect various kinds of locally relevant visual artifacts as the means by which they organize their online interaction. In synchronous computer-mediated communication systems such as these, actors produce an assortment of visual artifacts - textual and graphical - to achieve co-presence and establish indexical symmetry with respect to matters of relevant concern. The work that actors do when posting graphical and textual materials is the work of creating "readable" visual artifacts that allow recipients to achieve a sense of interaction by making sense of what they see in the chat system.

When it comes to talk, co-presence and the contiguity of actions provide for turn taking as the foundational organization of talk-in-interaction (Schegloff, 2007). In chat systems of the kind we have investigated, continguity is not a relevant or determining factor in assessing the meaning of an action. It is not about what just happened or what happens next. It is about the way that readers connect objects through reading's work to create a "thread of meaning" from the various postings available for inspection. Proximity may be more relevant to the sense making required in chat systems than contiguity. Chat systems are about posting objects for visual inspection that allow readers to make connections between these posted objects based on their availability for inspection and the features they display rather than on a strict notion of their position in a sequence. This means that sequentiality is not something that has to be built based on a notion of the contiguity of actions as in talk-in-interaction. Rather, reading's work in chat is precisely the process by which actors constitute a sequence of actions as interaction from the production and inspection of available visual artifacts.

The specific procedures by which readers and authors constitute interaction from the production and inspection of visual artifacts in chat have been described above. In chat, participants rely on the proximity rather than the contiguity of text posting and graphical objects as a way of achieving a sense of progression in their interaction. Specific lexical, grammatical and, in the case of graphical artifacts, graphical resources are used to link postings of various sorts, to demonstrate that postings are to be seen as linked and to display what that link consists of. In addition to using reference tools in the production of chat text, when available, to regulate one's own actions and the actions of others, actors indicate with the use of ellipses and other continuation markers (short and grammatically incomplete postings, etc.) that they are producing a series of postings that are to be read as a sequence, even though the postings may not be contiguous. When producing graphical objects in the whiteboard, actors use proximity and its achievement by moving objects within the whiteboard space to indicate they are producing the composite features of what is being produced as a single object. The temporal sequence of the production of whiteboard objects is not necessarily treated as a relevant consideration in the construction 
of whiteboard objects, whereas the locational proximity of these objects with respect to each other may be treated as relevant.

In chat environments, social interaction is the local achievement of reading's work, understood to be both the production and receipt of visual artifacts (both textual and graphical) that are designed to provide through their proper inspection adequate resources by which actors constitute:

- The presence of actors in the system,

- The co-presence of actors who are mutually orienting to each other and the actions they perform,

- The indexical ground of conditionally relevant objects and texts, and

- Indexical symmetry among participants with respect to these visual artifacts.

Online interaction using text chat is not an impoverished ("narrow bandwidth") version of talking, but a form of interaction with its own appropriate system of interactional practices. CSCL researchers wishing to analyze chats or to design environments to support chat should take into account the characteristics of chat interaction presented here.

\section{References}

Fuks, H., Pimentel, M., \& Pereira de Lucena, C. (2006). R-U-Typing-2-Me? Evolving a chat tool to increase understanding in learning activities. International Journal of Computer-Supported Collaborative Learning, 1(1), 117-142. Retrieved from http://dx.doi.org/10.1007/s11412-006-6845-3.

Garcia, A., \& Jacobs, J. B. (1998). The interactional organization of computer mediated communication in the college classroom. Qualitative Sociology, 21(3), 299-317.

Garcia, A., \& Jacobs, J. B. (1999). The eyes of the beholder: Understanding the turn-taking system in quasisynchronous computer-mediated communication. Research on Language and Social Interaction, 34(4), 337-367.

Goodwin, C. (2000). Action and embodiment within situated human interaction. Journal of Pragmatics, 32 , 1489-1522.

Hanks, W. (1992). The indexical ground of deictic reference. In C. Goodwin \& A. Duranti (Eds.), Rethinking context: Language as an interactive phenomenon. Cambridge, UK: Cambridge University Press.

Hanks, W. (1996). Language and communicative practices. Boulder, CO: Westview.

Hanks, W. F. (2000). Intertexts: Writings on language, utterance, and context. Lanham: Rowman \& Littlefield.

Herring, S. (1999). Interactional coherence in cmc. Journal of Computer Mediated Communication, 4(4). Retrieved from http://jemc.indiana.edu/vol4/issue4/herring.html.

Livingston, E. (1995). An anthropology of reading. Bloomington: IN: Indiana University Press.

Pollner, M. (1974). Mundane reasoning. Philosophy of the Social Sciences,, 4(35), 35-54.

Sacks, H., Schegloff, E. A., \& Jefferson, G. (1974). A simplest systematics for the organization of turn-taking for conversation. Language, 50(4), 696-735. Retrieved from www.jstor.org.

Sarmiento, J., \& Stahl, G. (2008). Extending the joint problem space: Time and sequence as essential features of knowledge building. Paper presented at the International Conference of the Learning Sciences (ICLS 2008), Utrecht, Netherlands. Retrieved from http://GerryStahl.net/pub/icls2008johann.pdf.

Schegloff, E. A. (2007). Sequence organization in interaction: A primer in conversation analysis. Cambridge, UK: Cambridge University Press.

Schönfeldt, J., \& Golato, A. (2003). Repair in chats: A conversation analytic approach. Research on Language and Social Interaction, 36(3), 241-284.

Stahl, G. (2006). Sustaining group cognition in a math chat environment. Research and Practice in Technology Enhanced Learning (RPTEL), 1(2), 85-113. Retrieved from http://GerryStahl.net/pub/rptel.pdf.

Stahl, G. (2008). Integrating synchronous and asynchronous support for group cognition in online collaborative learning. Paper presented at the International Conference of the Learning Sciences (ICLS 2008), Utrecht, Netherlands. Retrieved from http://GerryStahl.net/pub/icls2008.pdf.

Stahl, G. (Ed.). (2009). Studying virtual math teams. New York, NY: Springer. Computer-supported collaborative learning book series, vol 11 Retrieved from http://GerryStahl.net/vmt/book.

Suchman, L. A. (1990). Representing practice in cognitive science. In M. Lynch, Woolgar, S. (Ed.), Representation in scientific practice. Cambridge, MA: MIT Press.

Zhao, S. (2003). Toward a taxonomy of copresence. Presence; teleoperators and virtual environments, 12(5), 445-455.

Zhou, N., Zemel, A., \& Stahl, G. (2007). Information as a social achievement: Collaborative information behavior in CSCL. Paper presented at the international conference on Computer-Supported Collaborative Learning (CSCL '07), New Brunswick, NJ. Retrieved from http://GerryStahl.net/vmtwiki/nan.pdf. 